\title{
Symmetry boundary condition in dissipative particle dynamics
}

\author{
Souvik Pal ${ }^{1}$, Chuanjin Lan ${ }^{1}$, Zhen $\mathrm{Li}^{2}$, E. Daniel Hirleman ${ }^{1}$ and Yanbao $\mathrm{Ma}^{1 *}$ \\ ${ }^{1}$ School of Engineering, University of California, Merced, Merced, CA 95343, USA, \\ ${ }^{2}$ Division of Applied Mathematics, Brown University, Providence, RI 02912, USA
}

*Corresponding author

University of California, Merced

Science and Engineering Building, RM 381

5200 N. Lake Road

Merced, CA 95343

Tel.: +1 (209) 228-4046

Email:yma5@ucmerced.edu 


\begin{abstract}
Dissipative particle dynamics (DPD) is a coarse-grained particle method for modeling mesoscopic hydrodynamics. Most of the DPD simulations are carried out in 3D requiring remarkable computation time. For symmetric systems, this time can be reduced significantly by simulating only one half or one quarter of the systems. However, such simulations are not yet possible due to a lack of schemes to treat symmetric boundaries in DPD. In this study, we propose a numerical scheme for the implementation of the symmetric boundary condition in both dissipative particle dynamics (DPD) and multibody dissipative particle dynamics (MDPD) using a combined ghost particles and specular reflection (CGPSR) method. We validate our scheme in four different configurations. The results demonstrate that our scheme can accurately reproduce the system properties, such as velocity, density and meniscus shapes of a full system with numerical simulations of a substystem. Using a symmetric boundary condition for one half of the system, we demonstrate about 50\% computational time saving in both DPD and MDPD. This approach for symmetric boundary treatment can be also applied to other coarse-grained particle methods such as Brownian and Langevin Dynamics to significantly reduce computational time.
\end{abstract}

\title{
Keywords:
}

Symmetry, boundary condition, dissipative particle dynamics, mesoscale 


\section{Introduction}

Dissipative particle dynamics (DPD) is a mesoscopic particle based simulation technique proposed by Hoogerbrugge and Koelman in 1992 [1] which is extensively used to model behavior of complex fluids, especially at length scales between atomic and continuum scales [2]. In this regime, continuum theories often fail when molecule mean free paths are comparable to the characteristic transport length scales [3, 4], and atomistic simulation techniques, such as molecular dynamics (MD), become computationally prohibitive [5]. To tackle this problem, instead of tracking individual atoms or molecules, DPD tracks coarse-grained particles that are composed of a cluster of atoms or molecules. DPD has been successfully employed to model mesoscopic flows in broad fields including complex fluid suspensions[3, 8,9$]$, polymer melting and mixing [10-13], and biological systems, e.g., biological tissues [14] and DNA suspensions [15]. Even for classical fluid mechanics problems e.g., flow between parallel plates and flow in a channel, DPD results show good agreement with theoretical solution and CFD [16, 17].

One of the major factors determining the correctness of DPD simulation is the accurate implementation of physical boundary conditions. So far, significant research has been performed on the implementation of the commonly encountered solid wall boundary condition in flow systems. Usually, to model the wall, a layer of frozen DPD particles are placed at the position of the wall $[13,18-21]$. To prevent DPD particles to enter the wall, whenever a particle crosses the wall boundary, one of the 4 reflection schemes is implemented[18, 22, 23]: (a) specular (b) bounce forward (c) bounce back and (d) Maxwellian reflection. However, the literature on other boundary types for DPD is relatively sparse. 
A very common boundary condition for the simulation of fluid systems is symmetric boundary condition. The application of symmetric boundary condition (SBC) allows modeling only a part of the system, e.g., one half or one quarter, thus significantly reducing computer memory requirement and computational time. For instance, for a flow between two parallel plates, the resulting flow field is symmetric with respect to the central plane between the two plates. Similar symmetrical configurations can also be observed in channel flows and formation of liquid menisci. Mathematically, at such symmetric plane, the normal gradient of the quantity of interest becomes zero, i.e., $\frac{\partial \phi}{\partial n}=0$, where, $\phi$ denotes the variable of interest (pressure, velocity, density, concentration etc.) and $n$ denotes the normal direction to the plane. However, to the best of our knowledge, there is no systematic study on the implementation of symmetric boundary conditions in DPD except that Abu-Nada [24] employed just specular reflection scheme to model a symmetric flow field.

In this study, we present a first systematic study on the implementation of the SBC in DPD. By employing a layer or ghost image particles near the symmetry boundary, we thoroughly demonstrate the effectiveness and accuracy of our scheme in modeling symmetrical flow fields and sharp symmetric liquid menisci. For the validation of our scheme of the SBC, we simulate 4 standard cases: (1) flow through parallel plates [16], (2) flow through a channel [17], (3) a liquid meniscus between two parallel plates, and (4) an isolated spherical liquid droplet. The rest of the paper is organized as follows: in section (2) we provide a detailed explanation of the implementation of our SBC. In section (3) we discuss the results obtained from our simulations. In section (4) we analyze the savings in computation time obtained by simulating a 
reduced half or quarter system with SBC compared to a full system. Finally, in section (5) we discuss the possibilities of extending this method to other coarse grained particle based methods.

\section{Methodology}

\subsection{Dissipative particle dynamics formulation}

In DPP simulation, the motion of particles obeys Newton's second laws:

$$
\frac{d \vec{r}_{i}}{d t}=\vec{v}_{i}, \frac{d \vec{v}_{i}}{d t}=\vec{f}_{i}
$$

Where, $r_{i}$ and $v_{i}$ denotes the position and velocity vector respectively for the $i^{\text {th }}$ particle. The non-dimensional masses of all particles are set to one [2] in Eq. (1), so that the acceleration becomes equal to the magnitude of the force. The total pairwise force $\vec{F}_{i j}$ on the $i^{\text {th }}$ particle from the $j^{\text {th }}$ particle is computed by summing up contributions from conservative, dissipative and random components $\vec{F}_{i j}^{C}, \vec{F}_{i j}^{D}, \vec{F}_{i j}^{R}$, i.e., $\vec{F}_{i j}=\vec{F}_{i j}^{C}+\vec{F}_{i j}^{D}+\vec{F}_{i j}^{R}$. The total force on the $i^{\text {th }}$ particle, is obtained by summing up pairwise contributions as

$$
\vec{f}_{i}=\sum_{i \neq j} \vec{F}_{i j}=\sum_{i \neq j}\left(\vec{F}_{i j}^{C}+\vec{F}_{i j}^{D}+\vec{F}_{i j}^{R}\right)
$$

The summation is computed over particles which lie within a cutoff radius $r_{c}$ from the $i^{\text {th }}$ particle. We use the combined Verlet neighbor and linked cell list technique [6] to build the neighbor list where the particles within a radius $r_{v}$ is considered to be neighbors, such that $r_{v}>r_{c}$. The conservative force is computed from a soft repulsion potential, such that, 


$$
\vec{F}_{i j}{ }^{C}= \begin{cases}a_{i j}\left(1-\frac{r_{i j}}{r_{c}}\right) \hat{e}_{i j}, & r_{i j}<r_{c} \\ 0 . & r_{i j} \geq r_{c}\end{cases}
$$

Here, $a_{i j}$ is the maximum repulsion between particles $i$ and $j, r_{i j}=\left|\vec{r}_{i j}\right|, \vec{r}_{i j}=\vec{r}_{i}-\vec{r}_{j}, \hat{e}_{i j}=\frac{\vec{r}_{i j}}{r_{i j}}$. The dissipative force, which is responsible for the viscous effects in the system, is given by

$$
\vec{F}_{i j}^{D}=-\gamma \omega^{D}\left(r_{i j}\right)\left(\hat{e}_{i j} \bullet \vec{v}_{i j}\right) \hat{e}_{i j}
$$

The random force responsible for Brownian motion is given by

$$
\vec{F}_{i j}^{R}=\varphi \omega^{R}\left(r_{i j}\right) \theta_{i j}(\Delta t)^{-1 / 2} \hat{e}_{i j}
$$

In Eqs. (4) and (5), $\omega^{D}$ and $\omega^{R}$ are weight functions for dissipative and random forces respectively, $\vec{v}_{i j}=\vec{v}_{i}-\vec{v}_{j}, \gamma$ is the amplitude of dissipative force, $\varphi$ is the amplitude of the random force, and $\Delta t$ is the timestep. The term $\theta_{i j}$ is a randomly fluctuating variable that follows Gaussian statistics with zero mean and unit variance. As constrained by fluctuation-dissipation theorem, the weight functions $\omega^{D}$ and $\omega^{R}$ are related as [7]

$$
\omega^{D}(r)=\left[\omega^{R}(r)\right]^{2}, \quad \varphi^{2}=2 \gamma k_{B} T
$$

where $k_{B}$ is Boltzmann constant and $T$ is temperature. We choose $\gamma$ and $\varphi$ in Eq. (6) such that the $k_{B} T=1$. The weight functions are chosen as [2]

$$
\omega^{D}(r)=\left[\omega^{R}(r)\right]^{2}= \begin{cases}\left(1-\frac{r_{i j}}{r_{c}}\right)^{2}, & \left(r<r_{c}\right) \\ 0, & \left(r \geq r_{c}\right)\end{cases}
$$

The equations of motion are solved using a modified version of velocity-Verlet scheme [2]. 


\subsection{The symmetric boundary condition}

The symmetric boundary condition proposed herein is inspired from the conventional implementation of wall boundary condition that are modeled by frozen particle layers in conjunction with bounce-forward reflection based reintroduction scheme [25]. However, unlike the wall boundary, which is external to the system of DPD particles, a symmetric plane is an internal boundary and particles can cross the symmetry boundary. Therefore, we cannot use frozen fluid boundary particles to implement symmetric internal boundary. Thus, there are two key questions in the implementation of symmetric boundary condition: (1) how to construct a layer of particles collectively behaving as a "fluid boundary"? and (2) how do we evolve these "fluid boundary" particles without solving their equations of motion? The answers for these two questions are given below.

The implementation of a symmetric boundary is illustrated schematically in Fig. 1. Below the symmetric boundary, we consider a layer of ghost particles which are mirror images of the real fluid particles above the boundary. The regular DPD particles and ghost particles are represented by empty circles and solid black circles, respectively, in Fig. 1(a). This way, the particle distribution becomes exactly symmetric in a region on both side of the symmetric boundary which enforces the condition of symmetry. The layer of real fluid particles on top of the symmetry boundary is called real particle layer (RPL) while the layer containing its ghost images under the boundary is called the ghost particle layer (GPL). Due to the truncated nature of the potential, a real particle on the symmetry boundary can interact with particles within a radius $r_{v}$, as shown in Fig. 1(a). Therefore, the thickness of both RPL and GPL is $r_{v}$. The particles in the RPL interact with those in GPL through the same conservative force mentioned in Eqs. (3) or (8) and same dissipative and random forces mentioned in Eqs. (4) and (5). The 
GPL serves as fluid boundary which answers the first question raised in the above paragraph. The real particles in RPL are not allowed to cross the symmetry boundary in our scheme. Instead, they are reintroduced using a combined ghost particle and specular reflection (CGPSR) method.

The second question, regarding the evolution mechanism of the ghost particles, can be again solved using principle of symmetry. At each timestep, the coordinates of the ghost particles are updated in order to preserve the symmetry of RPL and GPL. This is achieved in two steps shown in Figs. 1(b) and (c). In step 1 (Fig. 1(b)), the new positions of real particles in the RPL are calculated after constructing the Verlet neighbor list. Let us consider a particle in RPL whose position is marked by point A and its ghost image, marked by point B in Fig. 1(b). If the plane of symmetry is $z=0$ plane, coordinates and velocity components of the particles at A and its image at $\mathrm{B}$ are related as

$$
\begin{aligned}
& x_{B}=x_{A} ; y_{B}=y_{A} ; z_{B}=-z_{A}, \\
& v_{x \_B}=v_{x \_A} ; v_{y_{\_} B}=v_{y_{\perp}} ; v_{z \_B}=-v_{z \_A} .
\end{aligned}
$$

At next time step, if the new position of particle A remains above the symmetric boundary, its new position lies at point $\mathrm{C}$ without any treatment.. However, if its new positon is $\mathrm{D}$, which is below the symmetric boundary (see Fig. 1b), the particle A is reintroduced back to the RPL at new position $\mathrm{C}$ using a specular reflection mechanism (i.e., $\mathrm{C}$ is the mirror image of $\mathrm{D}$ ). In step 2 (Fig. 1(c)), the ghost particle at B, which is the mirror image of $\mathrm{A}$, is moved to a new position E. . Here $\mathrm{E}$ is the mirror image of $\mathrm{C}$ The new position and velocity of the ghost particle is computed as

$$
\begin{aligned}
& x_{E}=x_{C} ; y_{E}=y_{C} ; z_{E}=-z_{C}, \\
& v_{x_{-} E}=v_{x_{-} C} ; v_{y_{-} E}=v_{y_{-} C} ; v_{z_{-} E}=-v_{z_{-} C} .
\end{aligned}
$$


Consequently, the particles in GPL always remain as mirror image of those in RPL even after integration and the symmetric boundary condition is perpetually maintained.

\section{Results and discussion}

\subsection{Case I: flow between two infinite parallel walls.}

The configuration of numerical simulations is shown in Fig. 2. The two parallel walls are positioned at $z= \pm 15.25$ as shown in Fig. 2(a). The boundary condition in $x$ and $y$ directions are periodic. The flow occurs in positive $x$ direction caused by applying a body force of magnitude 0.02 (in DPD units) along $x$. Fig. 2(c) shows the $y-z$ cross section. The half subsystem is shown in Fig. 2(b), where the symmetry boundary is at $z=0$ while the other boundaries are same compared to the full case in Fig. 1(a). Ghost particles are shown in Fig 2(b) and its $y-z$ cross section Fig. 2(d). The RPL and GPL are also shown in Fig. 2(d). For better visualization, a zoomed in section around the symmetry boundary is shown in Fig. 2(e). It is visually prominent that the fluid particles in RPL and ghost particles in GPL are mirror images of each other. The simulation parameters for this case are presented in Table 1.

\subsubsection{Velocity profile}

We compare velocity profiles of the half and full system along $z$ direction, i.e., $v_{x}(z)$ in Fig 3. Since the system is periodic in $x$ and $y$ directions, the velocity profile $\left(v_{x}(z)\right)$ will not differ along those directions. Therefore, $v_{x}(z)$ is obtained by spatially averaging over $x$ and $y$ direction as well as temporally averaging over 100 equally spaced snapshots during the final 20000 steps of total 110000 steps, enough to ensure a convergent velocity profile. The velocity profile for the full system is parabolic as expected and symmetric about $z=0$, i.e., $\left.\frac{\partial v_{x}(z)}{\partial z}\right|_{z=0}=0$. We perform two simulations for a reduced half system, one with CGPSR and 
another one with just specular reflection denoted by 'without ghost' in Fig. 3. CGPSR results in a a velocity profile which is qualitatively closer to the full profile compared to the one using just specular reflection. The error of CGPSR method in predicting velocity compared to the full system is only localized and maximum at the symmetry boundary. This error can be attributed to the artificial nature of CGPSR method. We also conduct quantitative comparison of $\left.v_{x}\right|_{z=0}$, i.e., $v_{\max }$ between the full and the two half cases. The theoretical parabolic velocity profile for the flow in between two parallel walls is given as:

$$
v(z)=v_{\max }\left[1-\left(\frac{z}{h}\right)^{2}\right], v_{\max }=\frac{\rho g h^{2}}{2 \mu},
$$

where, the viscosity $\mu=\frac{45 k_{B} T}{4 \pi \gamma r_{c}^{3}}+\frac{2 \pi \rho^{2} r_{c}^{2}}{1575}$ as prescribed by Groot and Warren [2]. This profile is also plotted in Fig. 4. The resulting value of $v_{\max }$ using the parameters in Table 1 is 8.63 while the values of $v_{\max }$ obtained from DPD simulations of the full and two half systems using CGPSR and specular reflection are $8.53,8.82$ and 9.18 respectively. Thus, the full system $v_{\max }$ obtained from our simulation differs only $\sim 1 \%$ from theoretical value clearly validating our DPD simulation code. It is to be noted that the expression $\mu=\frac{45 k_{B} T}{4 \pi \gamma r_{c}^{3}}+\frac{2 \pi \rho^{2} r_{c}^{2}}{1575}$ is an approximate estimation of viscosity as it was derived neglecting the contributions of conservative forces on viscosity of the fluid. Thus, we independently determine the viscosity for our DPD fluid corresponding to the set of parameters in Table 1 used in simulation case I using a technique called periodic Poiseuille flow (PPF). In this configuration, we apply periodic boundary conditions in all three directions and equal and opposite body forces $f$ in $x$ direction are applied as shown in Fig. 4 such that, $f=0.02$ for $0 \leq z \leq 15.25$, and $f=-0.02$ for $-15.25 \leq z<0$. The simulation is run for 160000 
steps and the velocity profile $u(z)$ is plotted in Fig. 4. The kinematic viscosity can be obtained by plotting the analytical expression $u(z)=\rho f z(d-|z|) / 2 \mu$ with various values of $\mu$ and identifying its value which results in closest match between the analytical plot and the simulated velocity profile. Here, $2 \mathrm{~d}$ denotes the width of simulation domain in $\mathrm{z}$ direction, i.e., for our case I, $d=15.25$. The analytical solution closest to the simulation corresponds to a $\mu=1.15$. Hence, the viscosity $\mu=1.08$ calculated from formula is only $6.5 \%$ different from $\mu=1.15$ that obtained from separate PPF simulation, again confirming the validity of our DPD code.

The $v_{\max }$ obtained using CGPSR method shows a closer agreement with the $v_{\max }$ for the full system (3.4\% difference) compared to the $v_{\max }$ with just specular reflection (7.62\% difference from full system). This is due to more realistic forces on the fluid particles close to the boundary in CGPSR due to the presence of ghost particle layer. The simulations in the rest of the paper for the half and quarter sub-systems are performed using CGPSR method.

\subsubsection{Density profile}

Next, we compare the density profiles along the $z$ direction for the full and the half system in Fig. 5. Similar to the velocity profile, density profile along $z$ direction, $\rho(z)$ is obtained by averaging over $x, y$ and time. The fluctuations in $\rho(z)$ close to the wall has been reported previously and several methods have been proposed to eliminate it $[16,26]$. However, wall boundary condition is not the focus of this paper. Instead, we focus on the density profile near symmetry boundary. It shows in Fig. 5 that $\rho(z)$ for half system matches closely with that for the full case, except near the symmetry boundary where the $\rho(z)$ suffers fluctuation due to the presence of SBC. However, the fluctuations are smaller in amplitude compared to the same near 
the wall boundaries. The reason for the smaller fluctuations is the fluidic nature of the GPL particles rather than frozen nature of wall particles.

\subsection{Case II: flow through a rectangular channel.}

The configuration of DPD simulation of flow through a rectangular channel is shown in Fig. 6. The flow takes place along the positive $x$ direction caused by applying a body force of magnitude 0.08 (in DPD units) along $x$. The channel has a $y-z$ cross section of $10 \times 10$, with a channel length along $x=50$ in DPD units. Periodic boundary condition is applied along $x$. The walls are modeled using the same approach as described in case I. The distribution of $v_{x}$ across the $y-z$ cross section is symmetric about both $z=0$ and $y=0$, given that the origin lies at the centroid of $y-z$ cross section. Therefore, we study two reduced subsystems, a reduced half system symmetric about $z=0$ shown in Fig. 6(b) and another reduced quarter system symmetric about both $z=0$ and $y=0$ shown in Fig. 6(c). For the system with quarter symmetry, the two separate GPLs along the left $(y=0)$ and the bottom $(z=0)$ symmetry boundary overlap at the bottom-left corner in Fig. 6(c). A zoomed in view of this overlapped corner is presented in Fig. 6(d). In Fig. 6(d), zone A is the part of the actual system with real fluid particles. Zones B and D are parts of left and bottom GPLs respectively. Zone C is the overlapped GPL, in which the ghost particles positions and velocities are related to the real particle position and velocities in zone A as,

$$
\begin{gathered}
x_{\text {zone C }}=x_{\text {zone A }} ; y_{\text {zone C }}=-y_{\text {zone A }} ; z_{\text {zone C }}=-z_{\text {zone A }}, \\
v_{x, \text { zone C }}=v_{x, \text { zone A }} ; v_{y, \text { zone C }}=-v_{y, \text { zone A }} ; v_{z, \text { zone C }}=-v_{z, \text { zone A }} .
\end{gathered}
$$

The simulation parameters are same as used in Case I. The contour of the magnitude of steady state velocity $v_{x}$ for the full, half and the quarter system is shown in Fig. 7. After running the 
simulation for 110000 steps, the contour is spatially averaged over $x$ direction and temporally averaged in a way similar to case I. The contours of half and quarter symmetry are both qualitatively and quantitatively consistent with that of the full system. As expected, all the contour lines are perpendicular to the line of symmetries. Also, the velocity at the origin is $\sim 2.55$ for the full channel in comparison with 2.51 for the half and 2.53 for the quarter channel. Hence, the implementation of SBC in reduced system successfully reproduces the velocity field consistent with its equivalent full system.

\subsection{Case III: A meniscus formed between two parallel plates}

In order to achieve a sharp meniscus interface, the conservative force needs to include both attractive and repulsive contributions as proposed by Warren [27] and is expressed as

$$
\vec{F}_{i j}^{C}=A_{i j} \omega_{c}\left(r_{i j}\right) \hat{e}_{i j}+B_{i j}\left(\bar{\rho}_{i}+\bar{\rho}_{j}\right) \omega_{d}\left(r_{i j}\right) \hat{e}_{i j},
$$

Where, $\omega_{c}\left(r_{i j}\right)=\left(1-r_{i j} / r_{c}\right), \omega_{d}\left(r_{i j}\right)=\left(1-r_{i j} / r_{d}\right), r_{d}=0.75 r_{c}[27], \bar{\rho}_{i}$ and $\bar{\rho}_{j}$ the local average densities at the position of particles $i$ and $j$ respectively and $A, B$ the amplitudes of the attractive and the repulsive part respectively. This formulation is known as multibody DPD (MDPD). The local density $\bar{\rho}_{i}$ is computed as $\bar{\rho}_{i}=\sum_{j \neq i} \omega_{\rho}\left(r_{i j}\right)$, where the sum runs over all particles $j$ within radius $r_{i j}<r_{d}$ and the weight function $\omega_{\rho}\left(r_{i j}\right)$ is expressed as [27]

$$
\omega_{\rho}(r)=\left\{\begin{array}{lr}
\frac{15}{2 \pi r^{3}}\left(1-r_{i j} / r_{d}\right)^{2},\left(r_{i j}<r_{d}\right) \\
0, & \left(r_{i j}<0\right)
\end{array} .\right.
$$

As illustrated in Fig. 8, a liquid meniscus formed between two parallel plates is simulated using the MDPD method. The MDPD simulation parameters are listed in Table 1. The distance 
between the two parallel plates is 20. Periodic boundary conditions are applied along $x$ and $y$ directions. Unlike the DPD systems discussed in previous two sections, the fluid does not flow here. Thus, velocity field is not a suitable basis for comparison of half and full systems. Instead, we compare meniscus shape to determine symmetry. The meniscus in this configuration is symmetric about $z=0$. The 3D isometric view of a snapshot of particle positions of full and the half system at steady state are shown in Figs. 8(a) and (b) respectively. The 2D projections onto $x-z$ plane are shown in Figs. 8(c) and (d) for the full and the half systems respectively. The RPL and GPL are marked in Fig. 8(d). Qualitatively, the shape of the half system is consistent with its full counterpart. For quantitative comparison, the contact angles $\theta_{1}, \theta_{2}$ and meniscus widths $d_{u}$ and $d_{l}$ as shown in Figs. 8(c) and (d) are extracted from numerical simulations. The existing meniscus shape detection strategies $[28,29]$ are only approximate and are often based on prior simplified assumptions about the meniscus shape [28]. Herein, we perform a completely different, more accurate 2D meniscus shape extraction. The extraction process is illustrated in Fig. 9. Considering the uniformity in y-direction, we take the average of meniscus shape along ydirection. Specifically, from a snapshot of particle positions at a particular timestep, we subdivide the meniscus in 4 slices along $y$ as shown in Fig. 9(a). For each of these slices, we project the particle positions onto $x-z$ plane and using Prewitt edge detection algorithm [30], extract the 2D shape as shown in Fig. 9(b). Next, we superimpose 4 of these shapes obtained from 4 slices of Fig. 9(a). The superimposed profiles are shown in Fig. 9(c) from which a spatially average shape is obtained marked by the black line. Finally, the angles $\theta_{1}$ and $\theta_{2}$ are computed using straight line fits on the spatially averaged profile. The simulation is run for 50000 steps, enough to ensure a convergent spatially average meniscus profile. The spatially averaged meniscus profile for the full and the half systems after 49000 steps are shown in Fig. 
9(d). The qualitative agreement between the two profiles is clearly evident from Fig. 9(d). We simulate systems with different meniscus shapes and contact angles by varying the solid liquid interaction parameter $A$ in Eq. (8). The quantitative comparison of the contact angles $\theta_{1}, \theta_{2}, d_{u}$, and $d_{l}$ between the full and the half systems are presented in Fig. 10. The different meniscus shapes are shown in the inset of Fig. 10(a). The error bars are computed from 10 time steps after reaching 49000 steps. As evident from Figs. 10(a), (b) (c) and (d), both the contact angles and the meniscus widths show same trend as the parameter $A$ in Eq. (11) changes. In addition, the average error between the half and full system values of $\theta_{1}, \theta_{2}, d_{u}$, and $d_{l}$ is $\sim 4.5 \%$, which demonstrates the validity of the implementation of CGPSR method for MDPD system.

We also compare the density distribution for the full and the half systems in Fig. 11. The density in the half system with SBC closely follows the distribution for the full system, except near the symmetry boundary, where the half system exhibits density fluctuation, similar to the trend shown in DPD cases (Fig. 5). The density fluctuations are small in amplitude compared the fluctuations near the wall, similar to DPD.

Similar to case I, we tested the implementation of SBC with just specular reflection without ghost particles. This approach resulted in the meniscus breakup and detachment from the symmetry boundary. At steady state, all the fluid attached to the upper wall in form of a single droplet and completely lost contact with the symmetry boundary which is completely unphysical and incorrect. This means that it is necessary to use ghost particles to reproduce the correct balance of attractive and repulsive forces at the symmetry boundary for MDPD simulations to capture the correct liquid meniscus shape.

\subsection{Case IV: An isolated spherical droplet}


An isolated liquid droplet, i.e., a droplet not in contact with any substrate, assumes a spherical shape in order to minimize surface energy. The sphere is a perfectly symmetric shape having infinite planes of symmetry passing through its origin. Herein, we simulate such a system where we consider just symmetry about $z=0$. The full and half system configurations are shown in Figs. 12(a) and (b) respectively. The full sphere lies in a simulation domain with $60 \times 60 \times 60$ in DPD units. The dimensions of the domain are significantly larger than the radius of the sphere ( 6.5). For the full system, $x$ and $y$ and $\mathrm{z}$ boundaries placed at \pm 30 are periodic (not shown in Fig. 12). For the half system in Fig. 12(b), dimensions and boundary conditions (periodic) along $x$ and $y$ are the same. However, the $z$ dimension is halved with $z=30$ and symmetric boundary at $z=0$. Similar to the full system, the top wall has no interactions with the sphere. The simulation parameters are same as those in case III. The simulations are run for 50000 steps. To extract a 2D shape out of the 3D spherical shape formed by particles, we follow the method outlined in previous section for determination of meniscus shape. However, instead of projecting the system slices only along $x-z$ plane, we project the $3 \mathrm{D}$ system onto a number of planes perpendicular to the $x-y$ plane as shown in Fig. 12(b). Each of these planes passes through the center of the sphere and is 15 degrees rotated from each other, spanning $360^{\circ}$, i.e., total 24 such planes. The 2D projection on one such plane, i.e., the $x-z$ plane, is shown in Fig. 12(c) along with the GPL. The extracted spatio-temporally averaged steady state 2D droplet shapes are compared in Fig. 12(d) where the solid and the dotted lines represent the half and the full system respectively. Again, we find excellent qualitative agreement between the shapes in Fig. 12(d). For quantitative validation, we compare the radii of the two profiles and check the angles formed by the profile with SBC with the symmetry boundary, i.e., $\theta_{1}$ and $\theta_{2}$ in Fig. 12(d) which for an ideal sphere should be $90^{\circ}$. The steady state radii of the simulated full and the half spheres 
computed by averaging over $r_{y}$ and $r_{x}$ (Fig. 12(d)) are 6.53 and 6.51 respectively, differing only by $\sim 0.4 \%$. Also the steady state values of angles $\theta_{1}$ and $\theta_{2}$ measured from the half system are $85^{\circ}$ and $87^{\circ}$. These numbers validate the correct implementation of SBC for the case of MDPD simulation of a spherical droplet.

\section{Computation time}

Our main objective for investigating the symmetric boundary condition is potential savings in computational time. In this section we analyze computation time required by full and symmetric subsystems. We also demonstrate how these time savings compare as system size changes. Each simulation is performed using a single core of Intel ${ }^{\circledR}$ Xeon ${ }^{\circledR}$ processor E5-2620 $(2.0 \mathrm{GHz})$ using Fortran programming language. The $\%$ breakup of time required by various activities in one integration step is shown in Fig. 13. The time saved by reducing system size $N_{P}$ will depend on how the time required by these activities depend on $N_{P}$. The neighbor list construction is $\sim \mathrm{O}\left(N_{P} \times\left(N_{P}-1\right)\right)$, i.e., $\sim O\left(N_{P}{ }^{2}\right)$. Using combined application of linked cell and Verlet neighbor list techniques this order is reduced to $\alpha$, such that $1<\alpha<2$. The time integration is $1^{\text {st }}$ order in $N_{P}$ i.e., $\sim O\left(N_{P}{ }^{1}\right)$ and the force computation is $\sim \mathrm{O}\left(N_{P} \times N_{N}\right)$, where $N_{N}$ is the average number of neighbors per particle. The time dependence of total computational time on $N_{P}$ will be a combination of these three orders, resulting in an order $\beta$, such that $1<\beta<2$, i.e., a non-linear

dependence on $N_{P}$. This means that by reducing the system size to $\frac{N_{P}}{2}$, more than twice speed up is possible for running the same number of timesteps. We represent the ratio of computational 
time for full and half systems by $\tau_{h}$ and the ratio of computational time for full and quarter systems by $\tau_{q}$.

The $\tau_{h}$ for case I and $\tau_{h}$ and $\tau_{q}$ for case II for various system sizes are plotted in Fig. 14. $\tau_{h}$ and $\tau_{q}$ are plotted along the primary and the secondary vertical axes respectively. As expected, the speedup ratio $\tau_{h}$ is indeed greater than two for all the system sizes for Case I and II. Similarly, for the quarter system in Case II, $\tau_{q}$ is more than four for all the system sizes. For both cases I and II, an increasing trend can be observed for both $\tau_{h}$ and $\tau_{q}$ as the system size increases. This means, with larger systems, simulating half or quarter subsystem will result in even bigger savings in time. The $\tau_{h}$ for cases III and IV (MDPD cases) are presented in Fig. 15. Unlike the increasing trend in Fig. 14, the $\tau_{h}$ for MDPD cases do not show any significant trend with increase in $N_{P}$. Also the speedup is a little less than two. This system size independence and values of $\tau_{h}$ smaller than that in cases I and II (standard DPD cases) can be attributed to relatively complex implementation of MDPD conservative force in Eq. (11) which requires, apart from neighbor list construction and force computation, an additional task, i.e., computation of local density.

\section{Extension to other particle based methods}

All three force components in Eq. (2) are pair wise additive requiring neighbor list construction. The key contribution of our scheme is to enable the construction of neighbor list for particles near the symmetric boundary using ghost particles thereby allowing simulating only one half or quarter. Therefore, the scheme can also be applied to other coarse grained particle based methods where the equation of motion for a particle requires calculation of pairwise additive forces from neighboring particles. The current popular particle based methods can be classified in three 
categories: (1) Microscopic (MD) (2) Mesoscopic (DPD, Brownian dynamics (BD), Langevin dynamics (LD)) and (3) Macroscopic (Discrete particle modelling (DPM)). Our implementation will be applicable for all of these simulations except MD due to the artificial nature of the ghost particles. The equation of motion for LD and BD has a form[31] very similar to DPD presented in Eq. (2) having the same three components, i.e., conservative dissipative and random. Our scheme will enable the calculation of the former two components which are pairwise additive. The calculation of random part near the symmetry is trivial. The forces involved in DPM simulations[32] are (1) fluid induced forces (2) contact forces (3) long range forces and (4) gravity. Of these, our scheme will enable calculation of the pairwise additive contact forces. However, calculation of other components near the symmetry boundary is not straightforward. We will report the extension of current scheme to these methods and compare relative gain in computational time in a future study.

\section{Conclusion}

A systematic study of the implementation of symmetric boundary condition (SBC) in DPD simulation is presented in this paper. The new method for the SBC in DPD simulations is tested in four cases, including two symmetric channel flows (Poiseuille flow between two fixed parallel plates and a symmetric flow in a rectangular channel), and two static liquid droplets (a meniscus between two parallel walls and a free droplet suspension without gravity). For both symmetric flows, the velocity profile in the half and quarter systems are correctly reproduced and consistent with the results of simulations with the full systems. For both static droplets, the liquid-vapor interface shape for the half system is correctly reproduced. For the meniscus, wall contact angles and meniscus widths near wall and at the symmetry boundary are in good agreement (within $\sim 4.5 \%$ ). For the simulation of spherical droplet, the radii for the full and half systems show 
excellent match (within $\sim 0.4 \%$ ). The angle of liquid-vapor interface at the symmetry boundary is $85^{\circ}-87^{\circ}$, which is close to ideal scenario, i.e., $90^{\circ}$. For DPD, implementation based on just specular reflection produces higher error in velocity at the symmetry boundary compared to the combined ghost particle and specular reflection approach. In MDPD simulations of meniscuses between two solid walls, specular reflection without ghost particles for the SBC produces completely wrong results, leading to detachment of meniscus from the symmetry boundary. These evidences firmly establish the importance of our combined approach.

For all systems, significant time savings are achieved. Reducing the system to half, more than twice speed up is achieved in DPD cases and the speed up increases with system size. In contrast, speed ups in MDPD cases are a little less than two and remain independent of system size due to relatively complex force calculation mechanism. The only downside of the current implementation is the density fluctuations near the symmetry boundary found in both DPD and MDPD simulations. The current symmetric boundary condition can be extended to other coarse grained particle methods such as BD, LD and DPM and we hope to communicate such implementations in form of a future study 


\section{Acknowledgement}

This work is supported in part by the United States Department of Energy under the ARPA-E program (DE-AR0000396) and by the California Energy Commission under Energy Innovations Small Grant Program (57652A/13-01TE). 


\section{Reference}

[1] P. Hoogerbrugge, J. Koelman, Simulating microscopic hydrodynamic phenomena with dissipative particle dynamics, EPL (Europhysics Letters), 19 (1992) 155.

[2] R.D. Groot, P.B. Warren, Dissipative particle dynamics: bridging the gap between atomistic and mesoscopic simulation, Journal of Chemical Physics, 107 (1997) 4423.

[3] X. Fan, N. Phan-Thien, N.T. Yong, X. Wu, D. Xu, Microchannel flow of a macromolecular suspension, Physics of Fluids (1994-present), 15 (2003) 11-21.

[4] G. Karniadakis, A. Beskok, M. Gad-el-Hak, Micro flows: fundamentals and simulation, Applied Mechanics Reviews, 55 (2002) 76.

[5] I.V. Pivkin, B. Caswell, G. Karniadakis, K. Lipkowitz, Dissipative particle dynamics, Reviews in Computational Chemistry, 27 (2010).

[6] D. Frenkel, B. Smit, Understanding molecular simulation: from algorithms to applications, Academic press, 2001.

[7] P. Espanol, P. Warren, Statistical mechanics of dissipative particle dynamics, EPL (Europhysics Letters), 30 (1995) 191.

[8] V. Symeonidis, G.E. Karniadakis, B. Caswell, A Seamless Approach to Multiscale Complex Fluid Simulation, Computing in Science \&amp; Engineering, 7 (2005) 39-46.

[9] E. Moeendarbary, T.Y. NG, M. Zangeneh, Dissipative particle dynamics: introduction, methodology and complex fluid applications - a review, International Journal of Applied Mechanics, 01 (2009) 737-763.

[10] A.G. Schlijper, P.J. Hoogerbrugge, C.W. Manke, Computer simulation of dilute polymer solutions with the dissipative particle dynamics method, Journal of Rheology (1978-present), 39 (1995) 567-579. 
[11] Y. Kong, C.W. Manke, W.G. Madden, A.G. Schlijper, Modeling the rheology of polymer solutions by dissipative particle dynamics, Tribology Letters, 3 (1997) 133-138.

[12] G. Pan, C.W. Manke, Developments toward simulation of entangled polymer melts by dissipative particle dynamics (DPD), International Journal of Modern Physics B, 17 (2003) 231235.

[13] Y. Kong, C.W. Manke, W.G. Madden, A.G. Schlijper, Simulation of a confined polymer in solution using the dissipative particle dynamics method, Int J Thermophys, 15 (1994) 10931101.

[14] B. Markus, P. Jacques, J. Jean-François, E. Jens, Dissipative particle dynamics simulations for biological tissues: rheology and competition, Physical Biology, 8 (2011) 026014.

[15] X. Fan, N. Phan-Thien, S. Chen, X. Wu, T. Yong Ng, Simulating flow of DNA suspension using dissipative particle dynamics, Physics of Fluids (1994-present), 18 (2006) -.

[16] I.V. Pivkin, G.E. Karniadakis, A new method to impose no-slip boundary conditions in dissipative particle dynamics, Journal of Computational Physics, 207 (2005) 114-128.

[17] E.E. Keaveny, I.V. Pivkin, M. Maxey, G. Em Karniadakis, A comparative study between dissipative particle dynamics and molecular dynamics for simple- and complex-geometry flows, The Journal of Chemical Physics, 123 (2005) -.

[18] M. Revenga, I. Zuniga, P. Espanol, I. Pagonabarraga, Boundary models in DPD, International Journal of Modern Physics C, 9 (1998) 1319-1328.

[19] P. Malfreyt, D. Tildesley, Dissipative particle dynamics simulations of grafted polymer chains between two walls, Langmuir, 16 (2000) 4732-4740.

[20] J.B. Gibson, K. Zhang, K. Chen, S. Chynoweth, C.W. Manke, Simulation of colloidpolymer systems using dissipative particle dynamics, Molecular Simulation, 23 (1999) 1-41. 
[21] J. Jones, J. áNoel Ruddock, N. Spenley, Dynamics of a drop at a liquid/solid interface in simple shear fields: a mesoscopic simulation study, Faraday Discussions, 112 (1999) 129-142.

[22] S.K. Ranjith, B. Patnaik, S. Vedantam, No-slip boundary condition in finite-size dissipative particle dynamics, Journal of Computational Physics, 232 (2013) 174-188.

[23] M. Revenga, I. Zuniga, P. Espanol, Boundary conditions in dissipative particle dynamics, Computer physics communications, 121 (1999) 309-311.

[24] E. Abu-Nada, Energy Conservative Dissipative Particle Dynamics Simulation of Natural Convection in Liquids, Journal of Heat Transfer, 133 (2011) 112502-112502.

[25] D.C. Visser, H.C.J. Hoefsloot, P.D. Iedema, Comprehensive boundary method for solid walls in dissipative particle dynamics, Journal of Computational Physics, 205 (2005) 626-639.

[26] I.V. Pivkin, G.E. Karniadakis, Controlling Density Fluctuations in Wall-Bounded Dissipative Particle Dynamics Systems, Physical Review Letters, 96 (2006) 206001.

[27] P. Warren, Vapor-liquid coexistence in many-body dissipative particle dynamics, Physical Review E, 68 (2003) 066702.

[28] B. Henrich, C. Cupelli, M. Moseler, M. Santer, An adhesive DPD wall model for dynamic wetting, EPL (Europhysics Letters), 80 (2007) 60004.

[29] C. Claudio, H. Björn, G. Thomas, Z. Roland, M. Michael, S. Mark, Dynamic capillary wetting studied with dissipative particle dynamics, New Journal of Physics, 10 (2008) 043009.

[30] J.M. Prewitt, Object enhancement and extraction, Picture processing and Psychopictorics, 10 (1970) 15-19.

[31] D.L. Ermak, J.A. McCammon, Brownian dynamics with hydrodynamic interactions, The Journal of Chemical Physics, 69 (1978) 1352-1360. 
[32] N.G. Deen, M. Van Sint Annaland, M.A. Van der Hoef, J.A.M. Kuipers, Review of discrete particle modeling of fluidized beds, Chemical Engineering Science, 62 (2007) 28-44.

[33] S. Chen, N. Phan-Thien, B.C. Khoo, X.J. Fan, Flow around spheres by dissipative particle dynamics, Physics of Fluids (1994-present), 18 (2006) -.

[34] P. Nikunen, I. Vattulainen, M. Karttunen, Reptational dynamics in dissipative particle dynamics simulations of polymer melts, Physical Review E, 75 (2007) 036713. 


\section{List of Tables:}

Table 1: The parameters used in DPD and MDPD simulations.

\section{List of Figures:}

Figure 1: The symmetric boundary condition implementation is schematically described. (a) The configuration of DPD particles and their ghost images are presented. The symmetry boundary layer (RPL) and ghost particle layer (GPL) are marked. (b) The specular reflection mechanism for updating fluid particle position is illustrated(c) the updating mechanism for ghost particle position.

Figure 2: The base case configuration of DPD simulation of flow in between two parallel plates is presented. (a) the isometric view of the snapshot of instantaneous particle positions for full system at the end of simulation. The flow takes place along positive $x$ axis. (b) the symmetric half system with SBC. The plane of symmetry is $z=0$. (c) 2D projection of the full 3D system of (a). The periodic boundaries along $y$ are shown. The three layer frozen wall particle layer is also shown. (d) the 2D projection of symmetric half system of (b) with SBC. The RPL and GPL are shown each having a width of $r_{v}$. (e) zoomed in view of a small region around the symmetry boundary. The mirror symmetry of fluid particles and ghost image particles are visually clear from this figure.

Figure 3: The variation of $v_{x}(z)$ with respect to $z$ is presented for three systems, (1) full system, (2)symmetric half system with SBC and (3) symmetric half system with just specular reflection based boundary at $z=0$ but using ghost particles. The analytical solution corresponding to the full system is also shown. The analytical solution is computed using Eq. (9). 
Figure 4: The simulated and analytical velocity profile $u(z)$ in periodic Poiseuille flow configuration is presented.

Figure 5: Density distribution with respect to $z$ is presented for the full system (black) and the symmetric half system with SBC (red). The green line is the distribution replicated from the other half.

Figure 6: The base case configuration of DPD simulation of flow through a rectangular channel. The velocity distribution in the $y-z$ plane has two symmetry planes, viz. $z=0$ and $y=0$. (a) the isometric view of the snapshot of instantaneous particle positions for full system at the end of simulation. The particle color scheme is same as in Fig. 2. (b) half symmetric system with respect to $z=0$ (c) quarter symmetric system with respect to both $z=0$ and $y=0$. (d) particle configuration near the corner is zoomed in for a better visualization. At the corner the GPL from the left and the bottom overlaps. The ghost particle positions in $\mathrm{C}$ are mirror images of those in zone $\mathrm{B}$ with respect to $z=0$ and mirror images of those in zone $\mathrm{D}$ with respect to $y=0$.

Figure 7: Contour of magnitude of velocity $v_{x}(y, z)$ is presented for the full, half and quarter symmetric systems.

Figure 8: The base case configuration of DPD simulation of liquid meniscus in between two parallel plates. (a) the isometric view of the snapshot of instantaneous particle positions for full system at the end of simulation.(b) the symmetric half system (c) the $2 \mathrm{D}$ projection of full 3D system onto $x-z$ plane. (d) the 2D projection of 3D symmetric half system onto $x-z$ plane. The angles $\theta_{1}$ and $\theta_{2}$.and meniscus width $d_{u}$ and $d_{l}$ are used for quantitative comparisons of full and half systems. 
Figure 9: (a) The 3D meniscus is subdivided into four slices along $y$. (b) for one such slice, a 2D meniscus shape projected onto $x-z$ plane is extracted using Prewitt edge detection algorithm[30] (c) Superposition of 4 such 2D shapes for four slices in Fig. 8(a). The superposed shapes are in blue. An average meniscus shape is obtained from them, as shown in black. The contact angles are obtained by fitting straight lines to this average shape. (d) The meniscus shapes for full and the half system are compared

Figure 10: Quantitative comparison of full and half systems in terms of angles (a) $\theta_{1}$, (b) $\theta_{2}$ (c) $d_{u}$ and (d) $d_{l}$ is presented. For the sake of clarity, error bars are not shown for (a) and (b) as they are very small $(\sim 2 \%)$. The error bars are computed over 10 timesteps after reaching steady state. In (c), the error bars corresponding to the half system data points are in blue while those corresponding to the full system are in red.

Figure 11: Density distribution along $z$ is presented. The density is obtained after spatially averaging along $y$ and temporally over 10 steps.

Figure 12: The base case configuration of DPD simulation of an isolated sphere is presented. (a) the snapshot of particle positions of the full system is shown. (b) The symmetrical half system is shown. To extract 2D shape, the 3D particle positions are projected onto a number of surfaces perpendicular to $x-y$ plane. Three of those planes are shown. These planes pass through origin and are at $15^{\circ}$ from each other, spanning $360^{\circ}$, i.e., total 24 planes. (c) 2D projected shape onto $x-z$ plane. (d) comparison of extracted average 2D shape for full and half systems. The average is obtained over projections onto 24 planes indicated in (b) as well as 10 timesteps after reaching steady state.

Figure 13: Break up of computational time required for various activities during one timestep. 
Figure 14: The ratio of computational times for running same number of timesteps is presented here with respect to various system sizes. Three sets are shown. The triangles represent the ratio of computational times of full and half systems ( $\tau_{h}$, primary axis) for case I, squares represent $\tau_{h}$ for case II and circles represent the ratio of computational times of full and quarter systems ( $\tau_{q}$, secondary axis) for case II.

Figure 15: The speed up time ratio $\tau_{h}$, for full over half systems with respect to $N_{P}$ in case III and IV are presented. 
Table 1: The DPD parameters

\begin{tabular}{ccc}
\hline & DPD & MDPD \\
\hline \hline$r_{c}$ & 1 & 1.5 \\
$r_{v}$ & 1.3 & 1.8 \\
$d t$ & 0.02 & 0.005 \\
$\rho_{\text {fluid }}$ & 4 & 6 \\
$\rho_{\text {wall }}$ & 4 & 6 \\
$a_{\text {fluid-fluid }}$ (Eq. 3) & $18.75[33]$ & - \\
$a_{\text {fluid-wall }}$ Eq. 3) & $9.68[33]$ & - \\
$\varphi_{\text {fluid-fluid }}$ (Eq. 5) & $3[34]$ & 6 \\
$\varphi_{\text {fluid-wall }}$ Eq. 5) & $3[34]$ & 6 \\
$\gamma_{\text {fluid-fluid }}$ (Eq. 4) & $4.5[34]$ & 18 \\
$\gamma_{\text {fluid-wall }}$ (Eq. 4) & $4.5[34]$ & 18 \\
$A_{\text {fluid-fluid }}$ (Eq. 8) & - & $-40[27]$ \\
$A_{\text {fluid-wall }}$ (Eq. 8) & - & $-13.7($ base \\
$B_{\text {fluid-fluid }}$ (Eq. 8) & - & case) \\
$B_{\text {fluid-wall }}$ (Eq. 8) & - & $25[27]$ \\
\end{tabular}




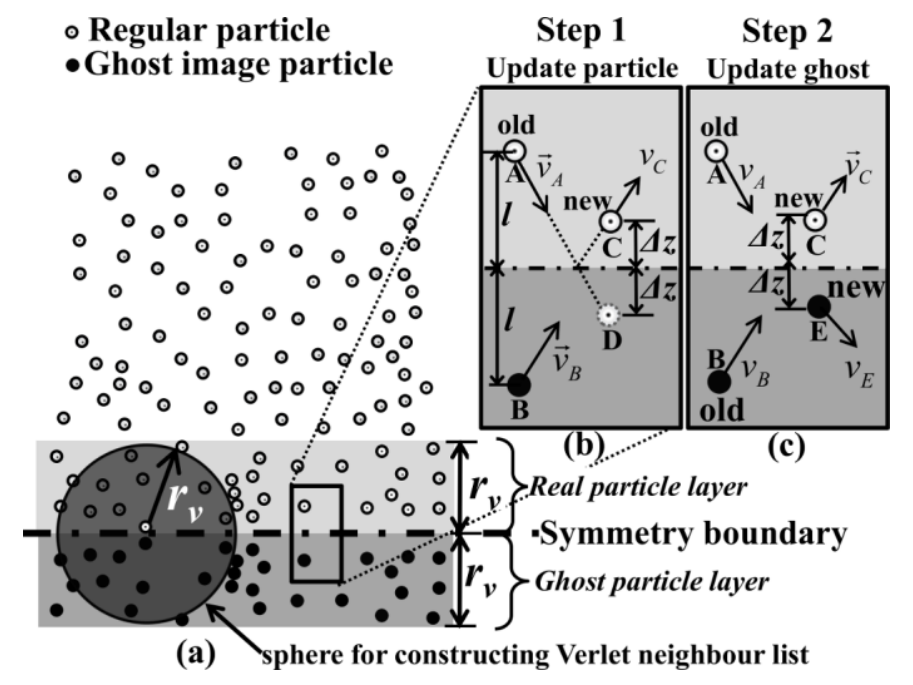

Fig. 1 


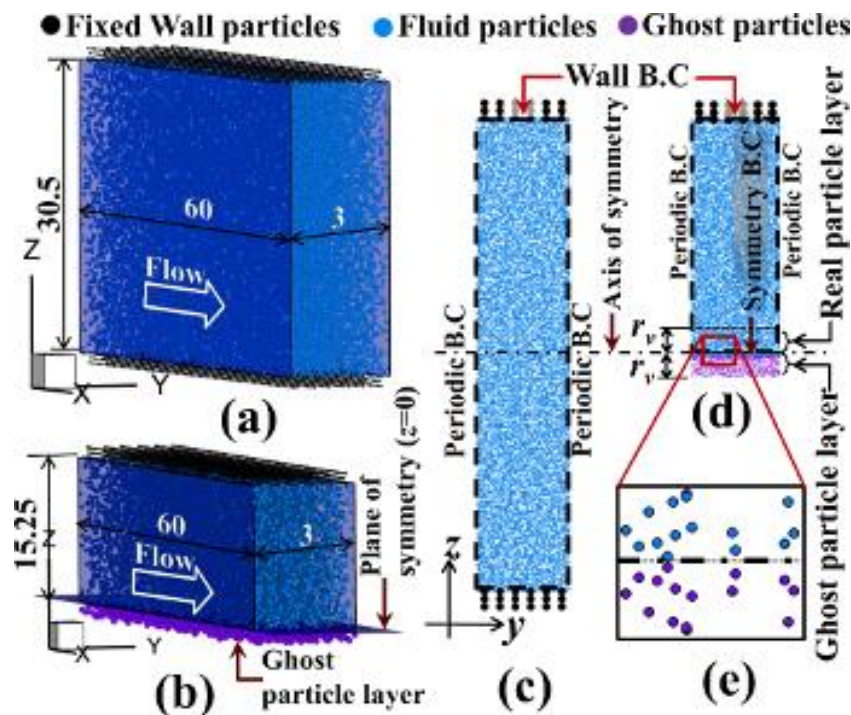

Fig. 2 


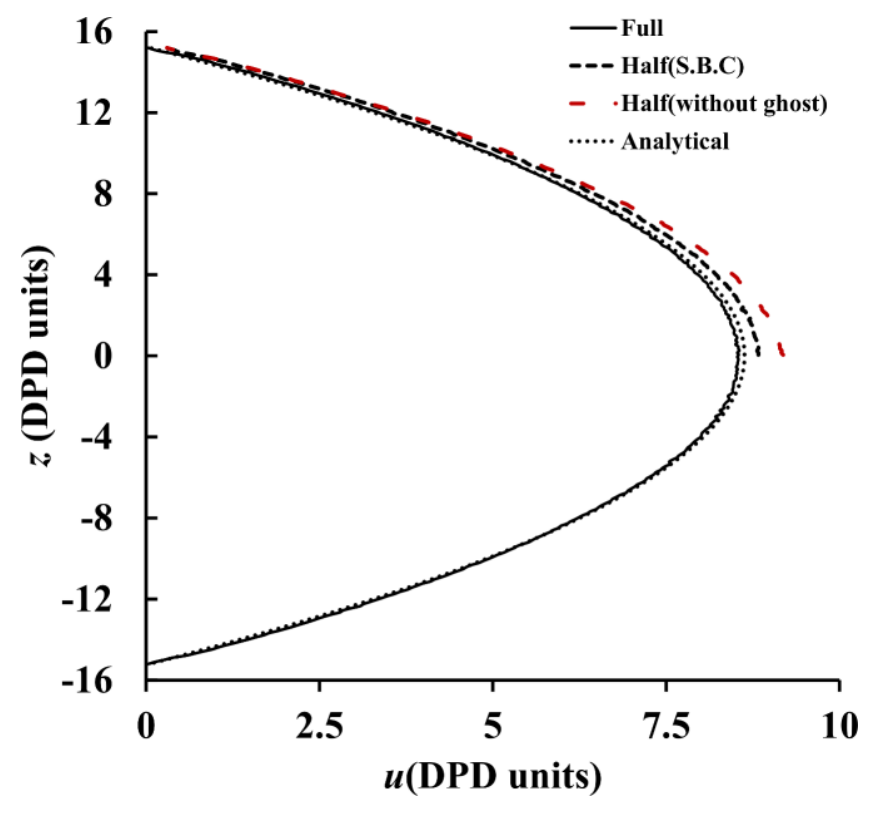

Fig. 3 


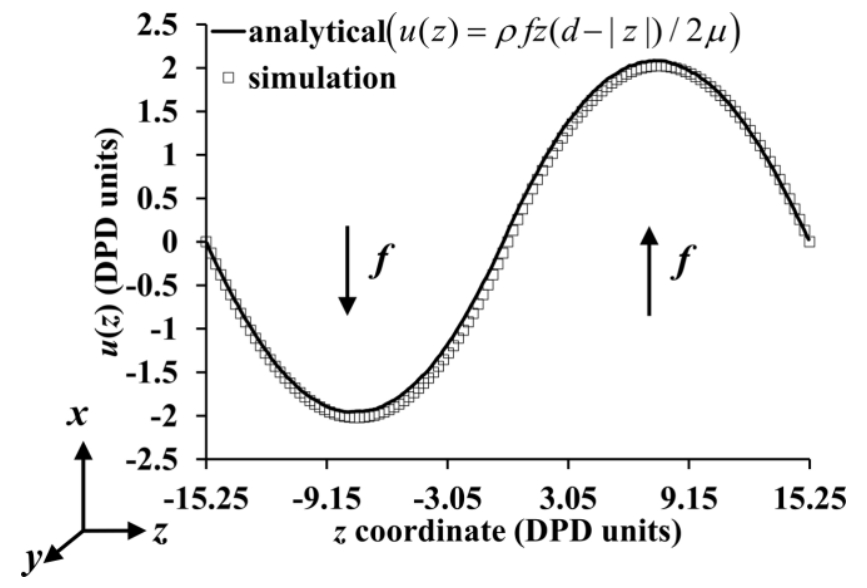

Fig. 5 


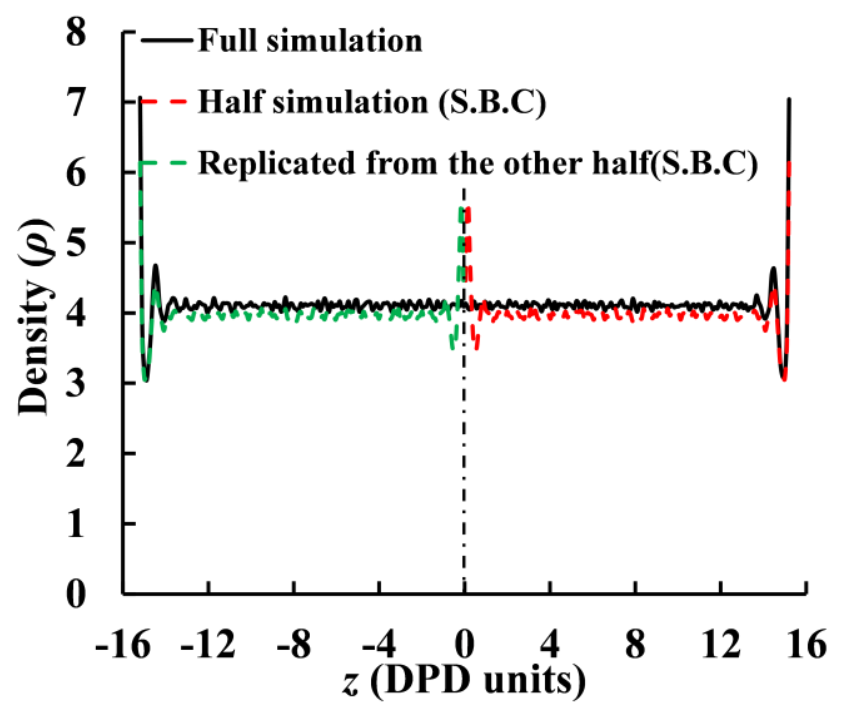

Fig. 5 


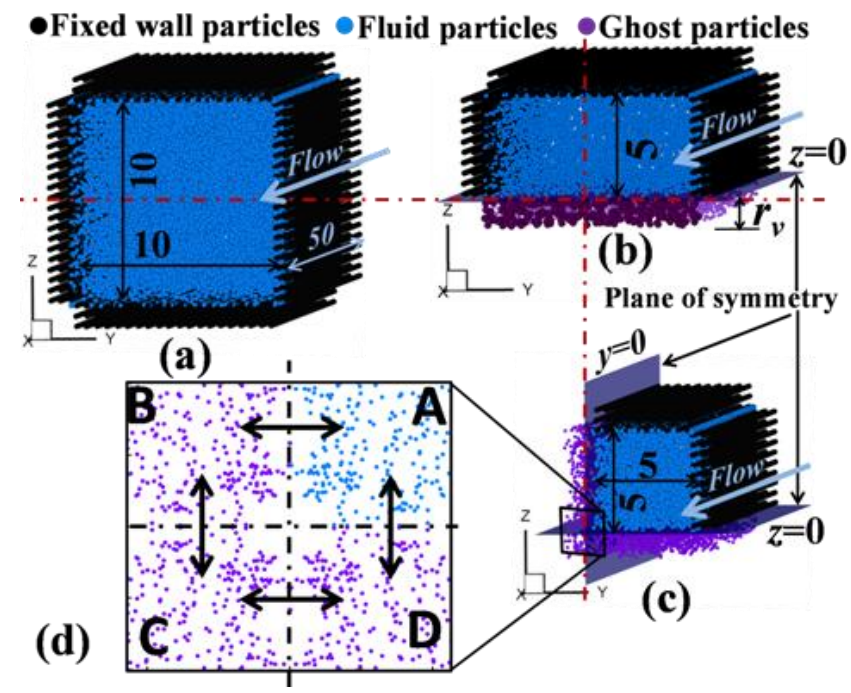

Fig. 6 


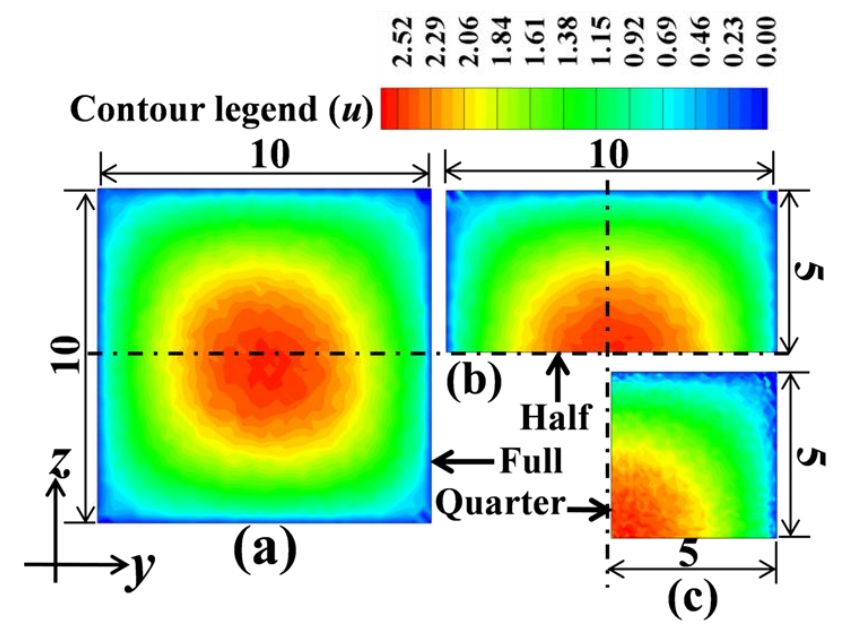

Fig. 7 


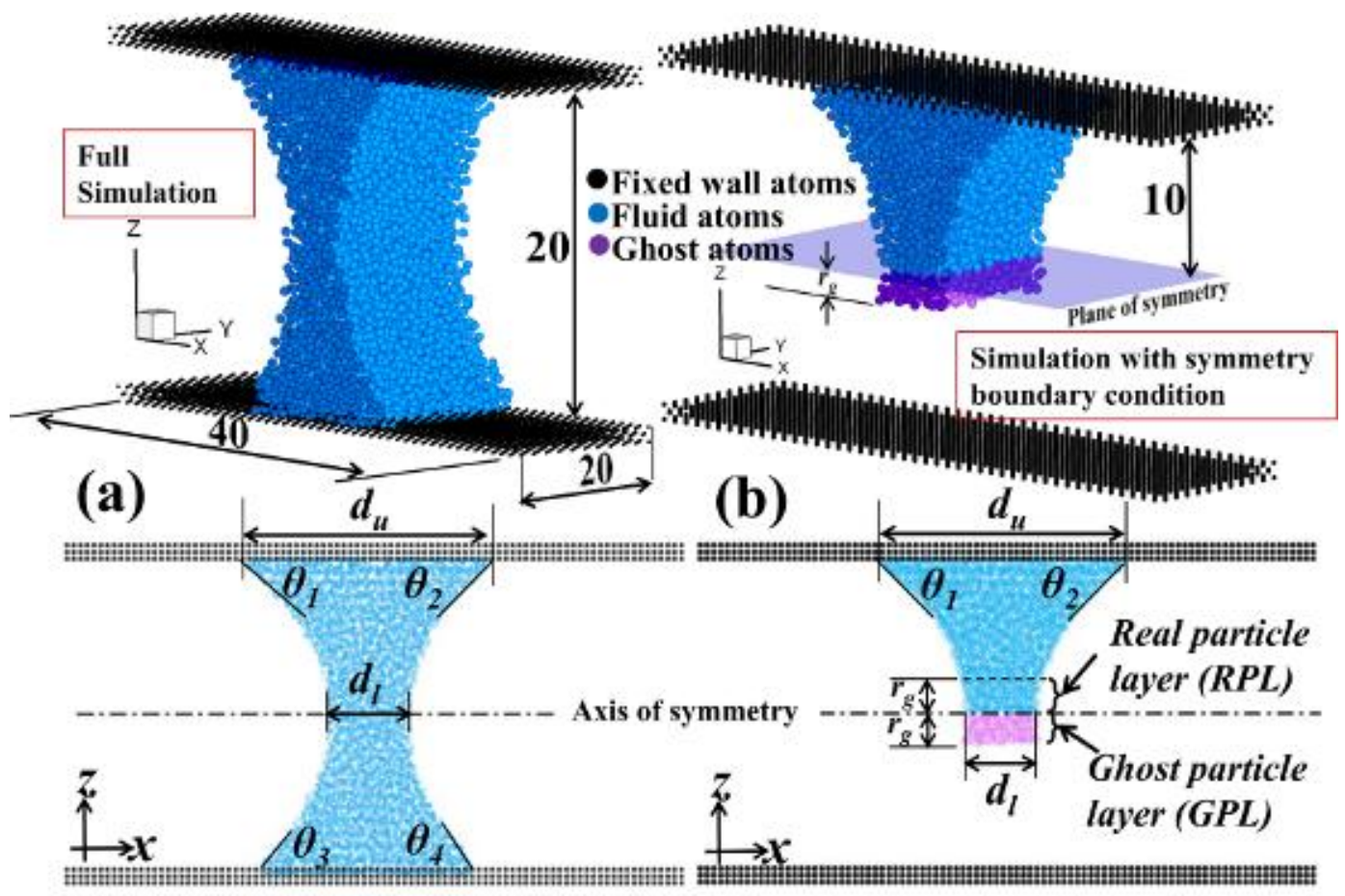

(c)

(d)

Fig. 8 


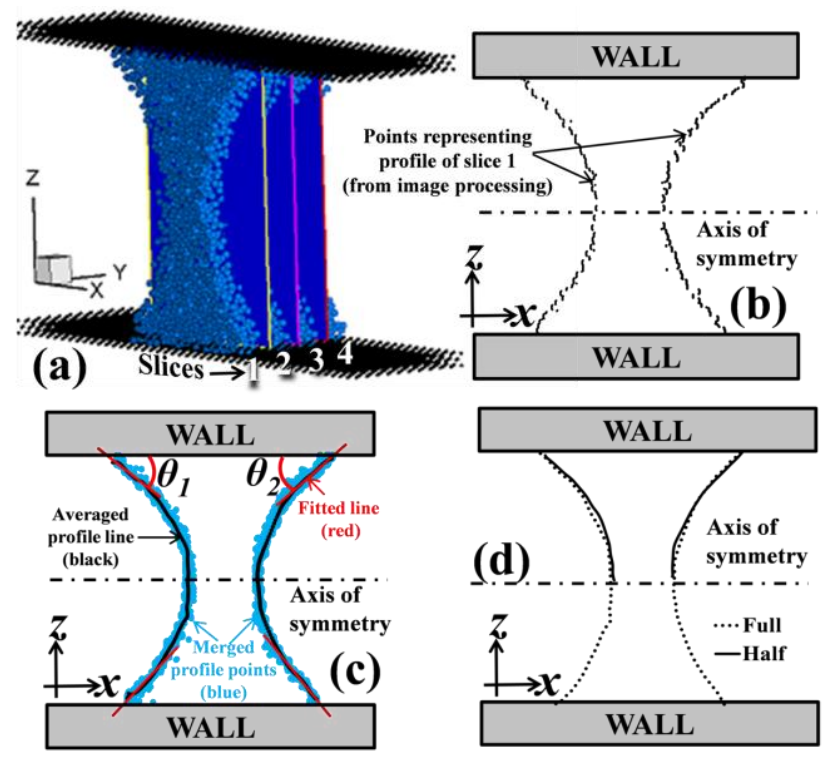

Fig. 9 


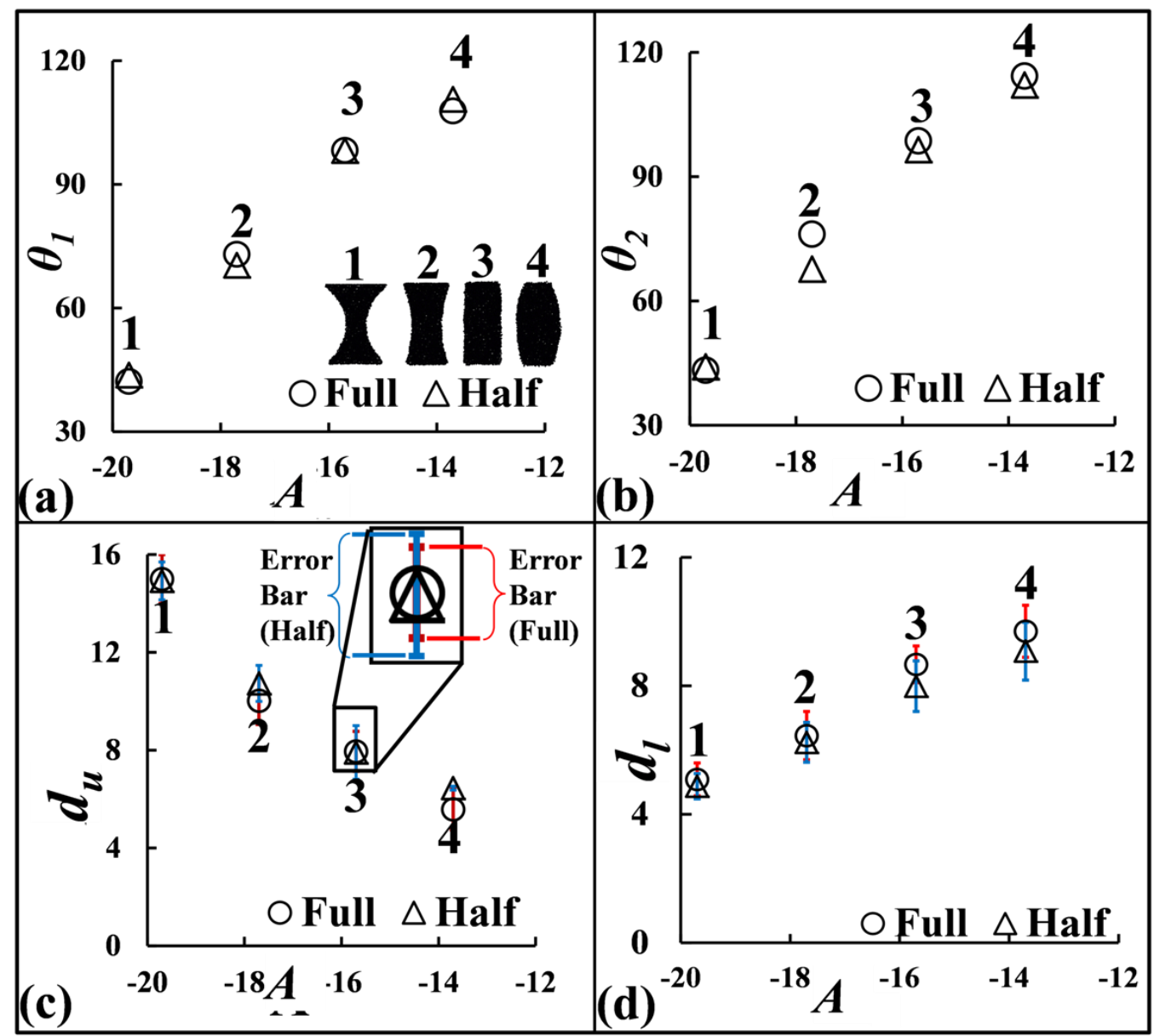

Fig. 10 


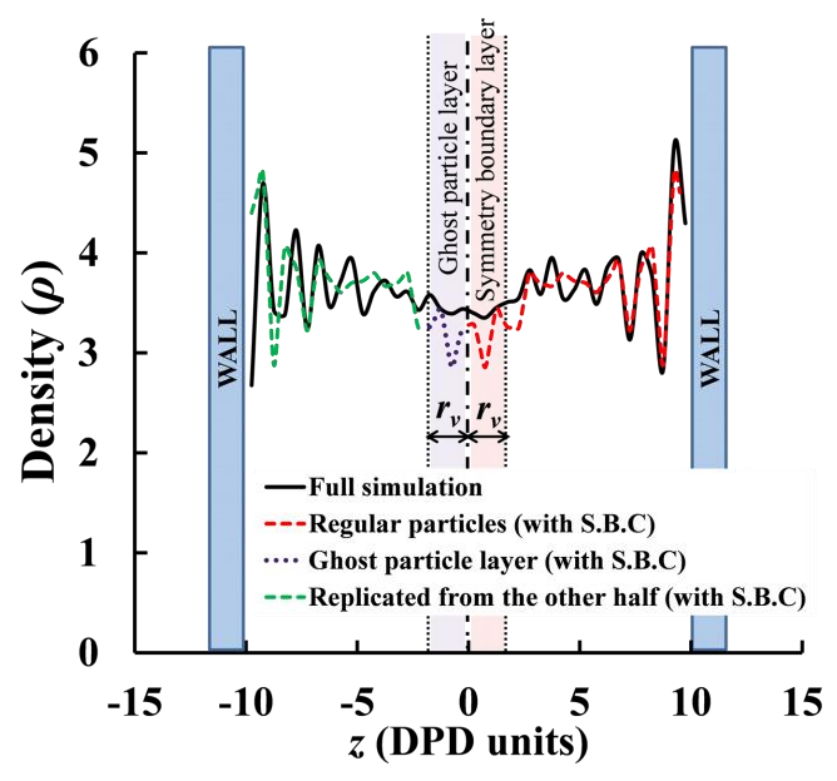

Fig. 11 


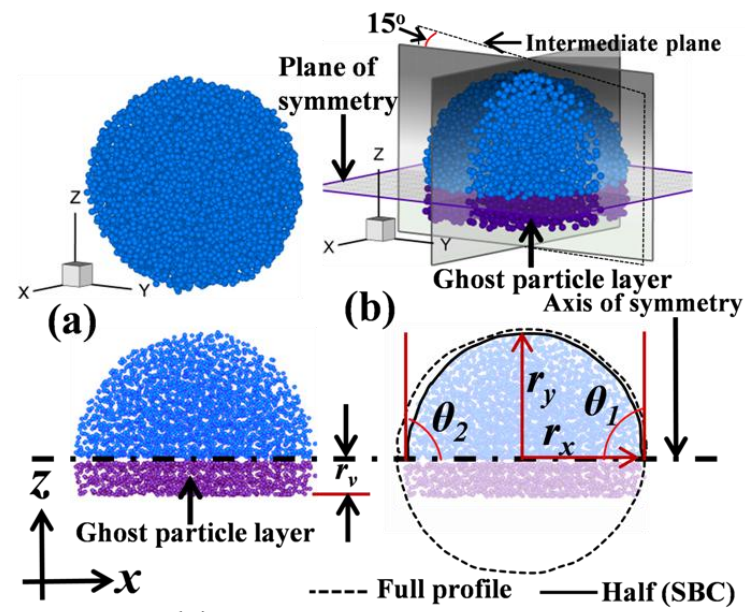

(c)

(d)

Fig. 12 


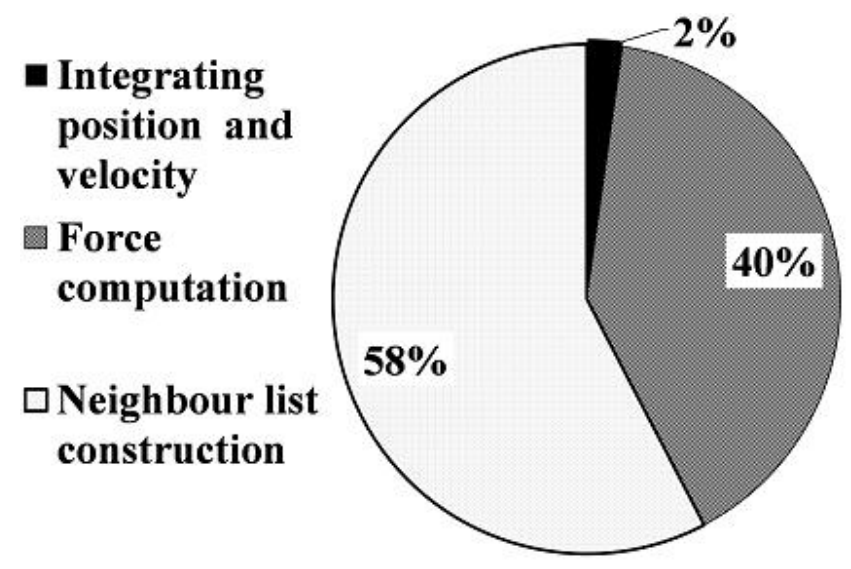

Fig. 13 


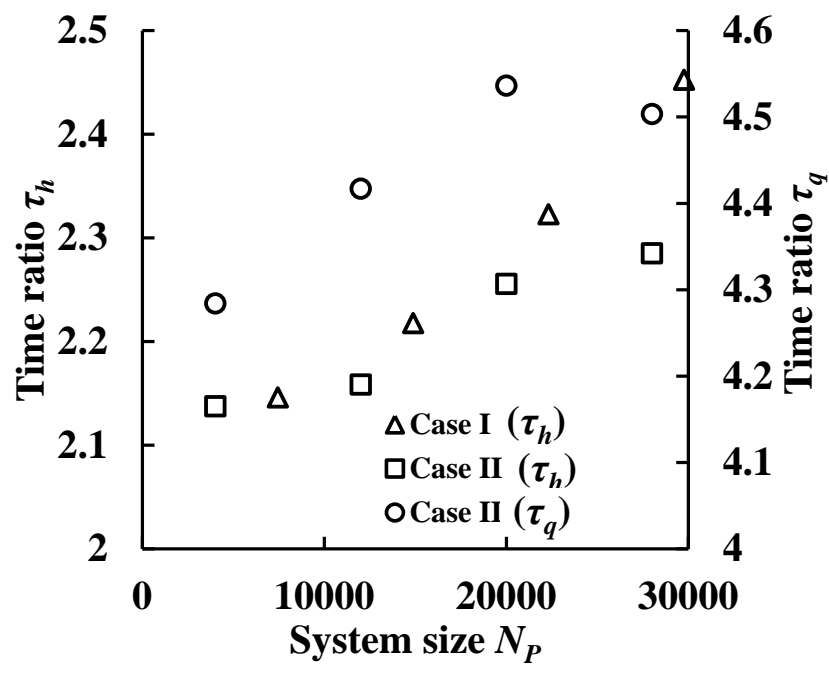

Fig. 14 


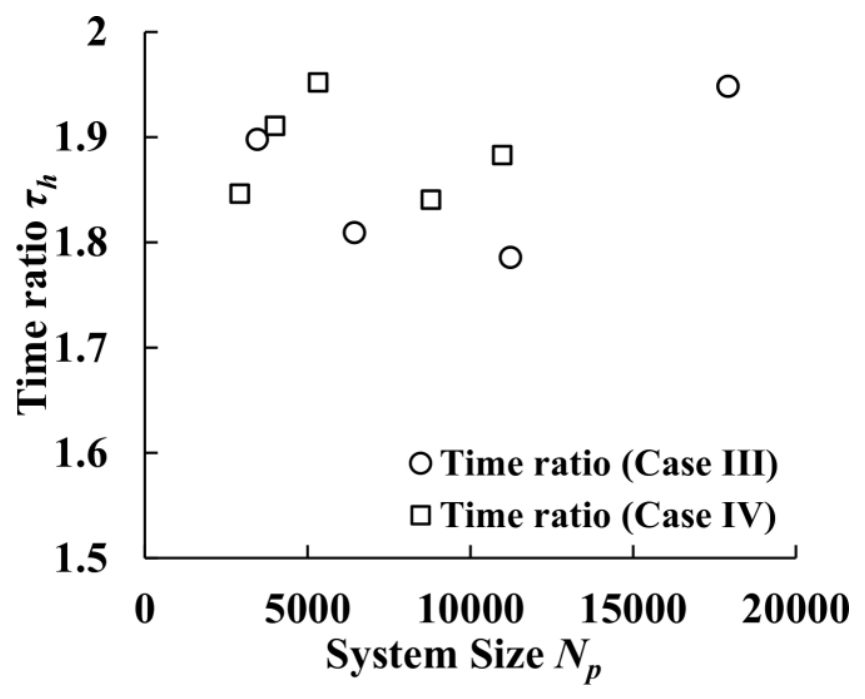

Fig. 15 